\title{
Supernova Remnants in the UWISH2 and UWIFE Surveys
}

\author{
Yong-Hyun Lee ${ }^{1}$, Bon-Chul Koo ${ }^{1}$ and Jae-Joon Lee ${ }^{2}$ \\ ${ }^{1}$ Department of Physics and Astronomy, Seoul National University, \\ 1 Gwanak-ro, Gwanak-gu, Seoul 151-742, Republic of Korea \\ email: yhlee@astro.snu.ac.kr, koo@astro.snu.ac.kr \\ ${ }^{2}$ Korea Astronomy and Space Science Institute, \\ 776, Daedeokdae-ro, Yuseong-gu, Daejeon, Republic of Korea \\ email: leejjoon@kasi.re.kr
}

\begin{abstract}
We report the preliminary results for the detection of $\mathrm{H}_{2}$ and [Fe II] line features around the Galactic supernova remnants (SNRs) from the UWISH2 and UWIFE surveys that cover the first galactic quadrant of $7^{\circ}<l<65^{\circ}$ and $|b|<1.3^{\circ}$. By this time, we have found a total of $17 \mathrm{H}_{2}$-emitting and 14 [Fe II]-emitting SNRs in the coverage, and more than a half of them are detected in both $\mathrm{H}_{2}$ and [Fe II] emissions, which implies that the environment of these SNRs might be complex and composed of multi-phase medium. In this paper, we present our identification strategy and some preliminary results including $\mathrm{H}_{2}$ and [Fe II] luminosity distributions.
\end{abstract}

Keywords. surveys, ISM: supernova remnants, infrared: ISM

\section{Introduction}

The UWISH2/UWIFE (UKIRT Wide-field Infrared Survey for $\mathrm{H}_{2} /[\mathrm{Fe}$ II] $)$ is nearinfrared (NIR) $\mathrm{H}_{2}-2.12 \mu \mathrm{m} /[\mathrm{Fe} \mathrm{II}]-1.64 \mu \mathrm{m}$ narrow-band imaging survey for the first Galactic quadrant $\left(7^{\circ}<l<65^{\circ} ;|b|<1.3^{\circ}\right)$ that have been performed with the WideField Camera at the United Kingdom Infrared Telescope (Froebrich et al. 2011; Lee et al., in prep.). Since the $\mathrm{H}_{2}$ and [Fe II] lines trace the shocked and/or fluorescently excited regions of molecular and atomic gases associated with jets/outflows around star formation regions, photo-dominated regions, planetary nebulae, evolved massive stars, and supernova remnants, the combination studies of the $\mathrm{H}_{2}$ and [Fe II] are one of the valuable tools for studying the formation and death of stars.

We have searched for the $\mathrm{H}_{2}$ and [Fe II] line features around the Galactic SNRs from the survey data. A total of $77 \mathrm{SNR}$ are falling in the survey area among the currently known 274 Galactic SNRs (Green 2009). These two complementary NIR $\mathrm{H}_{2}$ and [Fe II] imaging surveys can help us to understand not only the environment and evolution of the individual remnants but also statistical properties of the Galactic SNRs.

\section{Observation \& Continuum Subtraction}

The $\mathrm{H}_{2}$ survey had been completed in August 2011, while the [Fe II] survey is expected to finish in September 2013. The pixel scale of both surveys is $0.2 \operatorname{arcsec}$ by using $2 \times 2$ microstepping sequence, and the median seeings are $0.73 \operatorname{arcsec}$ and $0.80 \operatorname{arcsec}$ for $\mathrm{H}_{2}$ and [Fe II] surveys, respectively. The total per-pixel integration time is $720 \mathrm{~s}$ in both surveys, and the $5 \sigma$ detection limits of point sources in H- and K-bands are $\sim 19$ and $\sim 18$ magnitude, respectively. That limits may show diffuse structures as deep as the surface brightness of $\sim 10^{-19} \mathrm{~W} \mathrm{~m}^{-2} \operatorname{arcsec}^{-2}$. 

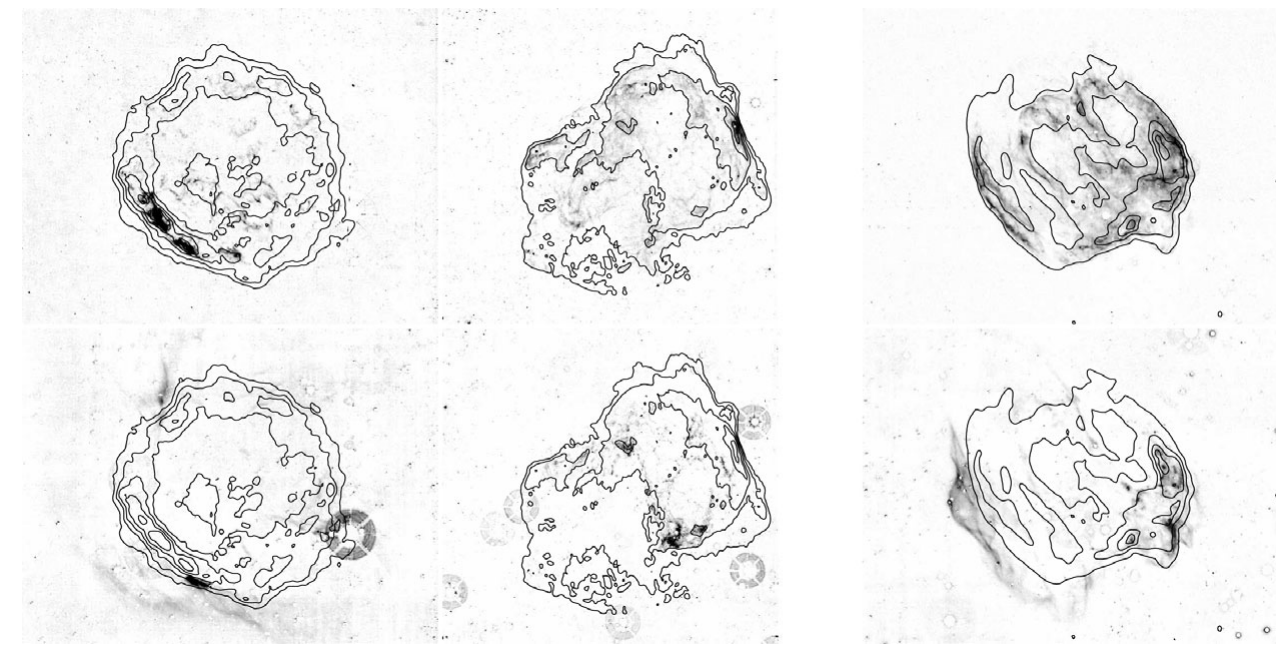

Figure 1. Continuum-subtracted images of G11.2-0.3 (left), 3C 391 (middle), and W49B (right). The upper-pannel shows [Fe II] in UWIFE, while the lower-pannel presents $\mathrm{H}_{2}$ in UWISH2. Radio continuum contours are superposed on each pannel.

In order to identify the narrow-band emission features, we subtract the $H$ and $K$-band continuum images obtained as part of the UKIDSS GPS (UKIRT Infrared Deep Sky Survey of the Galactic Plane) from the $\mathrm{H}_{2}$ and [Fe II] narrow-band images, respectively. For this, we developed an IDL-based automatic program for dealing with complex and space-variant Point Spread Functions (PSFs) using the subroutines of Starfinder code (Diolaiti et al. 2000).

\section{Preliminary Results}

By this time, we have found $17 \mathrm{H}_{2}$-emitting and 14 [Fe II]-emitting SNRs corresponding to $22 \%$ (17 out of 77 ) and $26 \%$ (14 out of 56 ) in detection rates, respectively. More than a half of them are newly-confirmed $\mathrm{H}_{2} /[\mathrm{Fe} \mathrm{II}]$-emitting SNRs that have never been reported in previous studies. This is likely to increase in future as we inspect the images in more detail. About $60 \%$ of the $\mathrm{H}_{2}$-emitting SNRs have [Fe II] features as well, thus the environment of these SNRs might be complex and composed of multi-phase medium.

Fig. 1 shows three SNRs (G11.2-0.3, 3C 391, and W49B) that radiate both strong $\mathrm{H}_{2}$ and $[\mathrm{Fe} \mathrm{II}]$ emissions. Note that the $[\mathrm{Fe}$ II] emission features are well correlated with the radio morphologies, while the $\mathrm{H}_{2}$ emissions are often found outside of the radio boundary.

According to our preliminary results, the extinction-corrected luminosities ranges from $2.4 \mathrm{~L}_{\odot}$ to $2.1 \times 10^{3} \mathrm{~L}_{\odot}$ in $\mathrm{H}_{2}$, and from $2.3 \mathrm{~L}_{\odot}$ to $8.6 \times 10^{3} \mathrm{~L}_{\odot}$ in [Fe II], and the brightest $\mathrm{SNR}$ is $\mathrm{W} 49 \mathrm{~B}$ in both $\mathrm{H}_{2}$ and [Fe II]. This luminosity range is comparable to that of the LMC SNRs, but slightly lower than that of the starburst galaxies, such as M82 and NGC 253 (Alonso-Herrero et al. 2003).

\section{References}

Alonso-Herrero, A., Rieke, G. H., Rieke, M. J., \& Kelly, D. M. 2003, AJ, 125, 1210

Diolaiti, E., Bendinelli, O., Bonaccini, D., et al. 2000, A\&AS, 147, 335

Froebrich, D., Davis, C. J., Ioannidis, G., et al. 2011, MNRAS, 413, 480

Green, D. A. 2009, Bulletin of the Astronomical Society of India, 37, 45 